\title{
Personnages illustres dans la monnaie tunisienne de 1956 jusqu'à 2010
}

Fonctions et apports

\section{Rim Yazidi}

\section{OpenEdition}

\section{Journals}

Édition électronique

URL : http://journals.openedition.org/communicationorganisation/4581

DOI : 10.4000/communicationorganisation.4581

ISSN : 1775-3546

\section{Éditeur}

Presses universitaires de Bordeaux

Édition imprimée

Date de publication : 1 juin 2014

Pagination : 193-208

ISBN : 978-2-86781-904-9

ISSN : $1168-5549$

\section{Référence électronique}

Rim Yazidi, «Personnages illustres dans la monnaie tunisienne de 1956 jusqu'à 2010 »,

Communication et organisation [En ligne], 45 | 2014, mis en ligne le 01 juin 2017, consulté le 19 avril

2019. URL : http://journals.openedition.org/communicationorganisation/4581; DOI : 10.4000/ communicationorganisation.4581 


\title{
Personnages illustres dans la monnaie tunisienne de 1956 jusqu'à 2010 fonctions et apports
}

\author{
Rim Yazidi ${ }^{1}$
}

\section{Introduction}

Les billets de banque sont des « objets » dont la fonction première est purement monétaire. Ils sont les moteurs des échanges entre les individus, ils permettent l'acquisition de biens et/ou de services. C'est leur valeur marchande qui compte avant tout. Émis par les banques centrales ou nationales, la monnaie fiduciaire, avec ses différentes valeurs, circule entre les individus en tant qu' « instrument financier».

L'aspect et l'esthétique de cette monnaie, c'est-à-dire la composition, le graphisme, etc. n'ont aucun rapport direct avec sa valeur marchande. Le billet ou la pièce vaut par son équivalent en marchandise ou service autant que par sa couleur ou les graphismes qu'il ou elle contient.

Nous savons, aussi, que la monnaie change, au fil du temps, en fonction de contraintes ou données économiques. Elle change non pas de valeur mais d'« apparence ». Donc un billet de banque ou une pièce de monnaie a une durée de vie déterminée en tant qu'objet d'échange, et le jour où ils perdent ce statut, on les retrouve comme des objets de collection, objets de musée.

$\mathrm{Si}$, d'une manière générale le design s'intéresse au côté esthétique de la chose, après avoir assuré sa bonne fonctionnalité, pour attirer l'attention, créer le besoin et enfin provoquer une action (l'attraction, le refus, l'achat, l'appropriation, etc.), « le design est devenu aujourd'hui un outil stratégique aussi important et puissant que le marketing ${ }^{2} . »$ On peut se demander, ici, quelle est la valeur rajoutée engendrée par le design ? Ne sommes-nous pas en droit de penser que les recherches au niveau du design, pour ce type de supports,

1 Inscrite en Doctorat en Sciences et Technologies du Design, Spécialité Design Image (2 a année 2012/2013, 2013/2014), à L'École Supérieure des Sciences et Technologies du Design (ESSTED), Denden, Tunis(ie). Enseignante contractuelle à l' ESSTED de 2011 à 2014.

2 BOUZIRI Imen, « Le design comme facteur de modernisation a-t-il une influence sur le comportement du consommateur tunisien ? ", in Les formes utilitaires en Tunisie au XXe siècle / sous la Dir. de Filali Aicha, Unité de recherche : Pratiques artistiques modernes en Tunisie, 2008, p. 14. 
ont d'autres objectifs et motivations ultérieures et non pas immédiates, qui seront en fin de compte d'en faire un objet de musée ?

Donc le billet de banque comme la pièce de monnaie n'ont-ils pas en réalité deux «vies » ? Une première quand ils sont en circulation et seule leur valeur monétaire compte, et une deuxième, où cette valeur devient nulle afin de laisser émerger le côté esthétique ? La monnaie devient importante par sa composition graphique et non plus par sa valeur marchande. Une composition qui implique des signes, des formes, des couleurs, qui s'articulent et d'où émerge forcément un sens, n'est-elle pas la cause essentielle et directe du fait que la monnaie devient « objet de musée » ? Et que son histoire se confond ainsi avec celle du pays, avec le progrès des techniques et avec l'évolution des goûts esthétiques?

Très peu de travaux de recherche, en Tunisie, se sont intéressés au côté graphique et conceptuel des billets de banque mis à part quelques mémoires de mastère (Belhassine, 2003 ; Guidara, 2004). La plupart des écrits et des ouvrages portent sur le caractère historique de la monnaie (ses débuts, son évolution à travers les civilisations, etc.) et le caractère économique (Lannoye, 2011 ; Khiri et Alexandropoulos, 2008 ; Khiri et Fenina, 2008).

Dans le cadre de cet article, l'accent sera essentiellement mis sur un seul élément iconique, à savoir les effigies des personnages illustres figurant sur les billets de banque de la Tunisie de 1956 jusqu'à 2010, période marquée par deux régimes politiques différents. Ces portraits seront l'axe central d'une étude graphique, puis d'une analyse sémiologique afin de relever les fonctions et les apports de leur présence sur les billets de banque. Ce choix est dicté par la position centrale et dominante, qu'ils occupent.

La première partie consiste en une approche graphique. Elle s'intéresse à l'« allure » générale des billets de banque, ou en d'autres termes, à la conception graphique. Le but de cette approche est de comprendre la raison pour laquelle on s'intéresse tant au côté esthétique de la monnaie, en particulier dans le cas de la monnaie tunisienne.

La deuxième partie consiste en une approche sémiologique des effigies de ces différents personnages illustres. Chacun de ces derniers renferme un sens symbolique que la sémiologie va déchiffrer en étudiant les différents aspects que présente le portrait. Le croisement des différents résultats issus des deux approches précédentes nous permettra de comprendre comment à travers ces personnages, la Tunisie commémore son patrimoine et glorifie son histoire et, au-delà, peut justifier l'intérêt du design pour un tel support.

\section{Les personnages comme moyen de différenciation}

Si on procède à une comparaison des billets de banque à travers le monde, au niveau de la gestion de la surface graphique, on constate qu'ils se ressemblent et obéissent à des règles « universelles », surtout en ce qui concerne les billets 
de banque comportant une effigie d'un personnage illustre, ce qui est le cas de la Tunisie.

Le billet de banque à travers le monde est généralement abordé à l'horizontale, ou plus exactement traité à "l'italienne ", à l'exception de quelques pays qui ont pris l'initiative de composer à la verticale, soit à la « française ». L'élément principal, qu'il soit personnage, monument, animal, etc. est fréquemment placé à droite de la composition. Certes, cette similitude entre les billets existe mais elle se limite au niveau de l'apparence. Du point de vue du contenu, c'est-à-dire des éléments graphiques mis en jeu, chaque pays se distingue des autres. Ainsi, il puise ses ressources dans son histoire, son patrimoine et son vécu. C'est ce qui crée une distinction au plan international. Une «stratégie de différenciation ${ }^{3}$ » (expression empruntée au marketing) est donc adoptée pour se distinguer des autres pays.

Toutefois, cette différenciation ne se limite pas qu'à ce niveau-là. En effet, même au niveau national on doit aussi adopter cette stratégie, puisque la logique de composition, les choix chromatiques, etc. sont les mêmes, surtout en ce qui concerne la monnaie appartenant à une même époque. Or, les billets de banque n'ont pas la même valeur monétaire. L'une des méthodes de différenciation est le choix du personnage illustre, mis en exergue, qui change d'un billet à un autre, qu'on ajoute à la différenciation par les couleurs. Donc personnage et couleurs sont les deux premiers signes distinctifs et éléments d'identité du billet.

Ainsi, en plus du fait que le design garantit à l'objet sa fonctionnalité en premier et une esthétique attractive en second, il lui offre aussi une particularité qui le différencie des objets similaires qui l'entourent. "Dans les entreprises, c'est à lui (design) que revient la mission d'élargir le champ de création de nouveaux produits, de sortir des sentiers battus, d'innover les manières de penser et de gérer l'identité des sociétés et des marques, auxquelles le consommateur s'identifie ${ }^{4} »$. Si on applique ceci au domaine de la numismatique, on trouve que les entreprises sont équivalentes aux pays émetteurs, les produits sont la monnaie et le consommateur, c'est le citoyen.

\section{L'euro en contre-exemple}

L'euro est la nouvelle monnaie commune des 27 pays membres de l'Union européenne. Concernant les billets de banque, la première constatation qu'on peut relever c'est qu'il y a absence d'effigies de personnages illustres. Avant cette union, la totalité des pays membres avait des portraits dans leurs billets qui évoquaient leur histoire tout en les distinguant les uns des autres en les rendant particuliers.

3 C'est une des stratégies marketing adoptée par les entreprises pour se distinguer de ses concurrents, surtout si le marché est saturé de produits ou services similaires.

4 Ibidem, p. 14. 


\section{$\mathrm{C} \& O \mathrm{n}^{\circ} 45$}

L'absence de ces personnages dans la monnaie de l'Union européenne actuelle (l'euro) s'explique par le fait qu'au moment de la mise en forme du projet, il y a eu désaccord sur ce sujet. Il fallait mettre des célébrités qui renvoient à l'Europe entière et non pas relatifs à une seule nation. Ceci était difficile voire impossible, car chaque personnage a servi son pays et non la totalité.

La solution était donc le recours à la carte de l'Europe et à la mise en valeur de sept monuments différents. Cependant, ces derniers n'ont jamais existé mais en les regardant ils donnent l'impression de « déjà vu ». Ces monuments sont relatifs à sept périodes clé de l'histoire de l'architecture : l'architecture classique, romaine, gothique, la renaissance, baroque et rococo, architecture verre et métal et architecture moderne. Donc ce sont des billets « anonymes ». De ce fait, l'un des rôles de la monnaie était perdu qui est la particularité et surtout la glorification de l'histoire nationale. Par contre, pour les pièces de monnaie, ils ont réservé une face européenne et une face nationale spécifique à chacun des pays membres. Une compensation, en quelque sorte.

\section{Approche graphique (au-delà de la différenciation)}

Il est évident donc que les compositions des billets de banque permettent de les identifier en tant que tels, ensuite les graphismes qui les composent facilitent la saisie de leurs valeurs monétaires et la reconnaissance du pays émetteur. La fonction première de la monnaie étant économique, elle autorise la circulation et les échanges continus entre les individus, les banques, les prestataires de services et autres. À ce niveau, c'est uniquement la valeur monétaire qui prédomine et elle est la seule à être prise en compte. Le design de la monnaie importe peu pour les usagers, il ne sert qu'à son identification (valeur, pays émetteur).

Seulement, il y a tout un travail de conceptualisation qui s'opère avant l'émission du billet, un travail qui suppose des choix motivés et qui a l'allure et la forme de n'importe quel support graphique de communication bidimensionnel. Une surface blanche qui grâce à des formes, à un fond va se construire en un tout harmonieux dépassant le simple état d'objet d'échange. En effet, cette surface est soumise à toutes les étapes de la chaîne graphique : de la conception jusqu'à l'impression et la finition, donc la mise en circulation. Ces étapes sont universelles et communes à tous les supports communicationnels.

L'étude des différents aspects graphiques des billets de banque tunisiens, a montré que les deux périodes historiques (l'ère de Bourguiba et l'ère de Ben Ali) sont très marquées graphiquement à tous les niveaux. Concernant la composition du support et la gestion de la surface graphique des billets de banque, l'époque de Bourguiba se caractérise par une composition variable, appliquant à chaque fois un gabarit de mise en page différent. 


\section{Exemples de gabarits de mise en page}

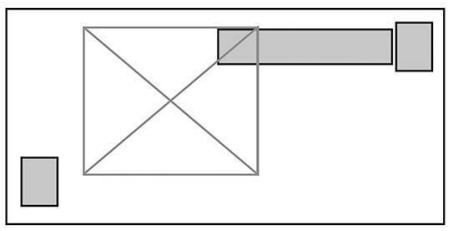

1973

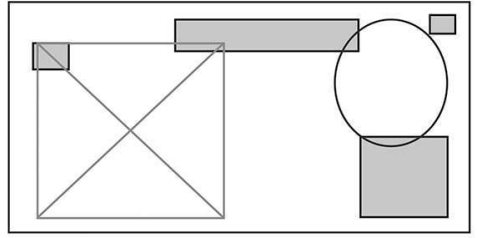

1983

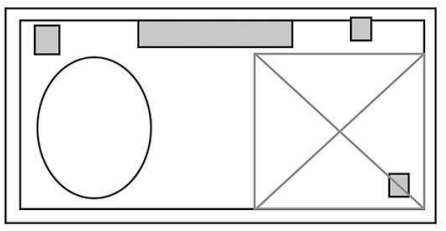

1980

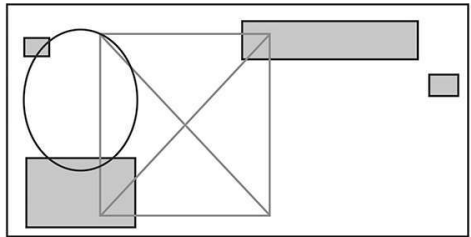

1986

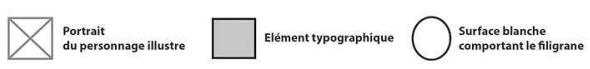

Figure 1: Exemples de gabarits de mise en page avant 1987 (Rim Yazidi)

Si on compare ces gabarits à d'autres gabarits de pays étrangers on remarque des similitudes.
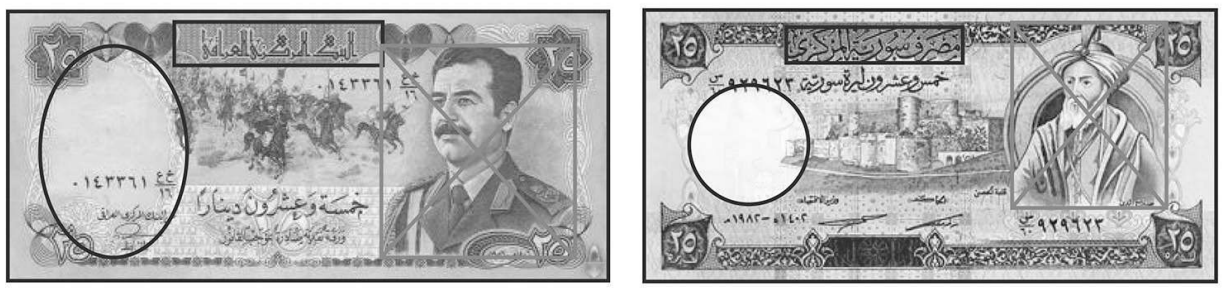

Figure 2: Adéquation des gabarits de mise en page avec les billets de banque de l'Iraq et de la Syrie. hitp://www.monnaiesdumonde.net/produits/produits.php?id_produit=336 http://www.monnaiesdumonde.net/produits/produits.php?id_produit=384

Pendant l'époque de Ben Ali l'allure générale des billets est presque la même, avec de légères variations à l'intérieur du rectangle d'empagement ${ }^{5}$. 


\section{Exemples de gabarits de mise en page}

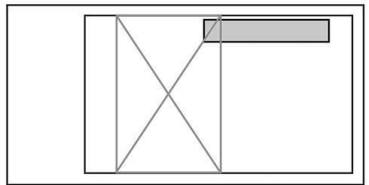

5 dinars

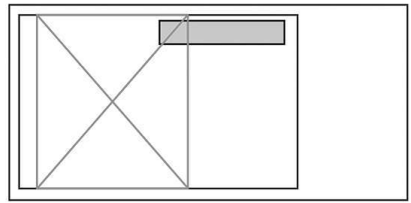

20 dinars

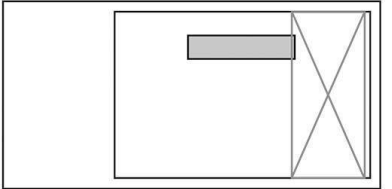

10 dinars (Didon)

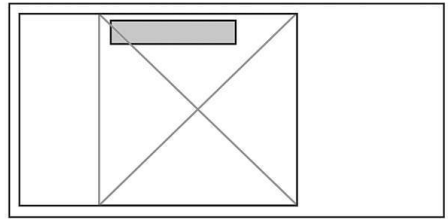

50 dinars

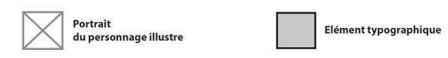

Figure 3: Exemples de gabarits de mise après 1987 (Rim Yazidi)

Pour les billets de cette période aussi, on relève une grande ressemblance avec ceux des autres pays étrangers. Cette ressemblance est notée au niveau de la division de l'espace.
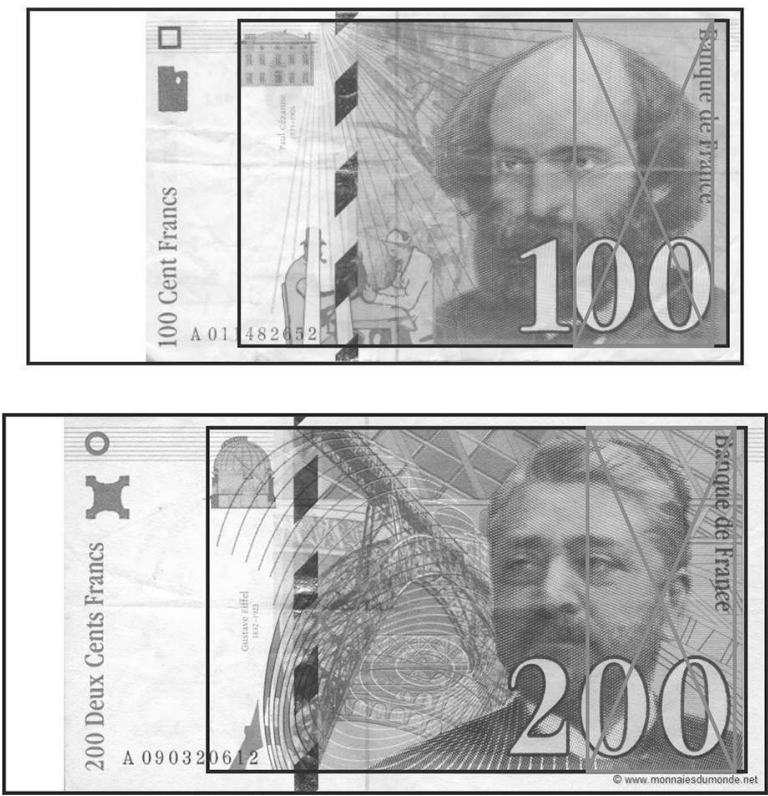

Figure 4: Adéquation des gabarits de mise en page avec les billets de banque de la France. http://www.monnaiesdumonde.net/produits/produits.php?id_produit=432

http://www.baudelet.net/monnaies/franc-france.htm 

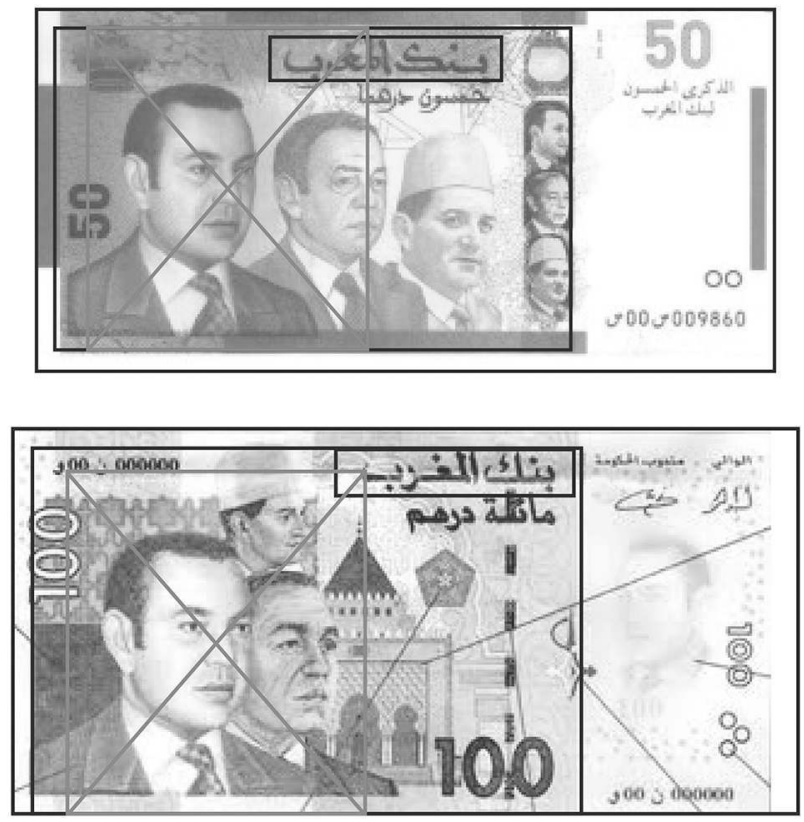

Figure 5: Adéquation des gabarits de mise en page

avec les billets de banque du Maroc. http://www.monnaiesdumonde.net/produits/produits. php?id_produit $=50$

Pour ce qui est du dessin, on remarque que la technique utilisée est la même, le traitement des valeurs à base de hachures qui traduisent l'effet de profondeur et de modelé ainsi que les ombres et lumières. Le nombre des hachures, leurs directions, la distance qui les séparent, créent une large gamme de valeurs de gris. Cependant, on est parfois face à des modifications de la forme de ces hachures qui se transforment en cercle ou en des traits discontinus.

Certes, les effigies de Bourguiba étaient réalisées par Hatem el Makki, qui était l'artiste responsable des dessins de la monnaie jusqu'aux années 90, alors qu'aujourd'hui on fait appel à d'autres dessinateurs, qu'ils soient professionnels ou encore au stade de l'apprentissage, qu'on choisit à la suite de concours. Au niveau de la couleur des billets de manière générale et des portraits en particulier, il y a une tendance à faire éclaircir les tons utilisés, si on compare la première période et la seconde.

Pour les éléments de sécurité, ils se sont multipliés d'une époque à une autre : sous Bourguiba il y avait seulement deux éléments de sécurité (le filigrane et le fil de sécurité), alors qu'aujourd'hui, on a atteint huit éléments différents (la taille douce, le filigrane, le fil de sécurité, l'image latente, les microlettres, l'encre à changement de couleur, les signes pour mal voyants en taille douce et la transvision). 


\section{$\mathrm{C} \& O \mathrm{n}^{\circ} 45$}

Pour la fabrication ou l'impression, elle se fait à la suite d'un appel d'offre lancé par la Tunisie au sein de certains pays européens comme l'Angleterre, la France et l'Allemagne. L'impression passe par deux étapes et deux procédés qui sont la lithographie et la taille douce avec un choix minutieux du papier fiduciaire.

Ainsi, on peut affirmer que la Tunisie obéit aussi aux règles « universelles ", relatives à la conception graphique de la monnaie. Cependant, elle est « unique » dans son contenu grâce aux choix des éléments iconographiques et typographiques mis en jeu, plus exactement grâce aux effigies des personnages illustres figurant dans les billets de banque.

C'est donc là où le billet de banque va prendre un statut qui justifie le travail de design, le statut d'un objet qui " raconte ", qui met en situation un personnage en relation avec des signes visuels témoins d'une certaine étape dans l'évolution économique ou culturelle du pays. Ce "statut " confère donc au billet de banque une valeur ajoutée qui n'a rien à voir avec sa valeur monétaire.

\section{Les personnages illustres retracent l'histoire du pays}

\section{Approche sémiologique}

Si l'on observe la totalité des billets de banque depuis l'indépendance jusqu'à aujourd'hui, on trouve qu'ils représentent différentes périodes de l'histoire de la Tunisie : l'antiquité est symbolisée par Didon, l'empire carthaginois est représenté à travers Hannibal, l'époque médiévale par Ibn Khaldoun ; quant à Kheireddine Pacha, Abou el Kacem Chebbi et Bourguiba, ils font référence à l'époque moderne.

La monnaie, avec tous les thèmes et les symboles qu'elle renferme, forme un condensé tangible de quelques fragments de notre histoire. Didon a fondé Carthage. Hannibal a élargi et a développé le territoire carthaginois, Ibn Rachik était un grand homme de lettres, Ibn Khaldoun était un sociologue très réputé par son attention au mode de vie des civilisations et de l'Homme en général. Kheireddine Ettounsi était un homme politique, avant-gardiste, son souci majeur était de faire avancer son pays et de le mettre sur la voie du progrès. Chebbi était un poète, qui a vécu pendant le colonialisme. À travers ses poèmes, il a soutenu son peuple en extériorisant ses maux. Bourguiba était un homme politique, un combattant qui a arraché l'indépendance de la Tunisie. Ainsi, chacun de ces personnages a marqué l'histoire à sa manière. Le fait de représenter des effigies de personnages illustres décédés et absents de nos jours, montre qu'on est reconnaissants et fiers d'avoir dans notre héritage culturel, des hommes et des femmes qui ont participé à la construction de la Tunisie. La représentation de l'ensemble de ces figures historiques tend à faire revivre leurs souvenirs et à perdurer dans la mémoire des citoyens. 
Comme on l'a remarqué précédemment, les portraits figurant dans la monnaie à travers le monde sont conformes, similaires et traités presque de la même manière au niveau de la technique utilisée, du traitement des valeurs à bases d'hachures ainsi que du choix du gros plan comme cadrage. La Tunisie a respecté ces "règles » dans une certaine limite. Durant toute la période d'après l'indépendance jusqu'à 1986, il n'y avait que le portrait d'Habib Bourguiba dans la monnaie. Cette présence continue, trouve sa justification dans le «culte de la personne " qui s'est développé dans le pays au fil des années. Bourguiba était le héros, le libérateur à qui le titre de « combattant suprême " a été attribué, pour signifier que ses combats continuent après l'indépendance. Ce qui est à noter, c'est que les portraits représentés sont réels, sans stylisation, et ont respecté la chronologie naturelle, en changeant avec l'âge et l'apparition des signes de vieillesse flagrants au niveau du visage et de la chevelure du personnage.

Cependant, après le changement du 7 Novembre 1987, les concepteurs des billets ont exploité d'autres méthodes dans la réalisation des portraits. Tout d'abord au niveau des cadrages, on est face à une diversité de plans : le gros plan (Didon, Hannibal, Ibn Khaldoun, Ibn Rachik et Bourguiba), le plan de moyen (Kheireddine Pacha, et Abou el Kacem Chebbi) et le plan d'ensemble avec des vues de profil, de face, de $3 / 4$ et en plongée qui ont chacune une signification particulière.

On a relevé aussi une stylisation ou une nouvelle interprétation de certains portraits de personnages, citons le cas d'Ibn Khaldoun où on a emprunté un style graphique bien particulier qui est celui des temps des pharaons. "Les Égyptiens adoptent un système conventionnel de représentation : dessin contour des surfaces colorées, visages de profil mais yeux de face pour insister sur l'essentiel ${ }^{6} »$.

On peut relever toutes ces caractéristiques dans l'effigie d'Ibn Khaldoun. Ce style cache différentes connotations bien spécifiques tels que l'immortalité et le pouvoir qu'on a associé auparavant aux pharaons. Ces connotations sont maintenant « léguées » à ce personnage illustre. Peut-être est-ce grâce à son ouvrage el Muqqadima qui est considéré comme un ouvrage immortel qu'il a mérité cette représentation... Ou bien peut-être est-ce dû au fait qu'Ibn Khaldoun a occupé un poste honorable en Égypte « Grand Cadi Malékite » et qu'il y est resté jusqu'à sa mort.

Le choix du portrait équestre de Kheireddine Pacha, est aussi novateur. Cette "innovation " ou ce " dépassement " des règles est au service d'un message bien précis qui valorise le personnage. En effet, la mise en scène avec tous ses détails : personnage sur un cheval blanc «Blanc, mais d'une blancheur éclatante, le cheval est le symbole de la majesté. Il est le plus souvent monté par celui qui est nommé Fidèle et Véritable ${ }^{7}$ ", un costume militaire, etc.

6 COCULA B. et PEYROUTET C., Sémantique de l'image pour une approche méthodique des messages visuels, Delagrave p. 65.

7 CHEVALIER J. et GHEERBRANT A., Dictionnaire des symboles, Robert Laffont / Jupiter, Paris, 1982, p. 231. 


\section{$\mathrm{C} \& O \mathrm{n}^{\circ} 45$}

convergeant vers une seule direction qui est la glorification. Cette mise en scène est réservée depuis la nuit des temps à des hommes de pouvoir pour les honorer.

Pour Hannibal, les éléments graphiques (personnage, monument et couleur) se complètent et convergent tous vers une même direction qui est la glorification de Carthage. Chebbi, même après sa mort, est resté proche des Tunisiens. On a souvent recours à ses poèmes et à ses vers. Ses mots sont contemporains à n'importe quel espace-temps. Sa présence sur le billet de banque de 30 dinars qu'on manipulait chaque jour et son portrait représenté de face, ces choix n'étaient pas fortuits. L'atout de cet angle de vue, c'est qu'il interpelle l'observateur. On avait l'impression qu'à chaque fois qu'on regardait ce billet, le personnage nous disait quelque chose. La couleur brune reflète le mal-être qu'il éprouvait. À côté de son portrait, on trouvait des monuments et des architectures symbolisant le progrès. La représentation de ces derniers est légitime vu que les écrits de Chebbi c'est-à-dire ses poèmes ont un caractère innovateur qui rompt avec le passé.

Didon, malgré son importance historique, graphiquement, on l'a dévalorisé par le choix d'un angle de vue en plongée qui, symboliquement, attribue au sujet représenté des connotations négatives d'écrasement. «On suggérera très efficacement l'idée d'infériorité, de défaite, d'effondrement psychologique, d'abattement physique ou moral du personnage ${ }^{8}$ ». La taille du portrait nuit aussi au personnage et à ce qu'il représente. Pour le billet de 10 dinars, on sent que l'accent a été plutôt mis sur la mosquée. La relation entre cette effigie et ce monument ne peut être expliquée que géographiquement : Elissa est la fondatrice de Carthage et la mosquée est située à cet endroit aussi. Le personnage n'a aucune relation ni avec l'islam ni avec la religion de manière générale.

Ibn Rachiq el Kairaouani, malgré son importance dans l'histoire de la Tunisie et son nom qu'on trouve jusqu'à aujourd'hui associé à des établissements éducatifs et culturels, on remarque qu'il y a une absence totale de références iconographiques à son sujet.

Ainsi, ces personnages font partie de notre héritage historique et culturel. Tous ont participé à la construction de la Tunisie. Leur présence dans les billets de banque peut être considérée comme une sorte de reconnaissance à leur égard.

Cependant, il faut noter que contrairement à l'époque Bourguiba, la chronologie n'a pas été respectée. Nous trouvons Abou El Kacem Chebbi sur un billet de 1997 et Didon sur un billet de 2005. Donc à partir de 1992 ce n'est plus la chronologie historique qui comptait mais la symbolique qui colle au personnage qui devient primordiale.

8 DUC, L'art de la composition et du cadrage: Peinture-Photographie-Bande Dessinée-Publicité, Fleurus Idées, Paris, 1993, p. 78. 


\section{Synthèse}

\section{Dualité entre histoire et modernité}

\begin{tabular}{|c|c|c|}
\hline Billets de banque & Personnages illustres & Autres éléments \\
\hline Billets de 1956 jusqu’à 1992 & $\begin{array}{l}\text { Habib Bourguiba } \\
\text { (Le combattant suprême, } \\
\text { le libérateur, puis le } \\
\text { constructeur innovateur) }\end{array}$ & $\begin{array}{l}\text {-L'amphithéâtre d'El Jem } \\
\text {-La grande mosquée de } \\
\text { Kairouan } \\
\text {-Collège Sadiki } \\
\text {-L'olivier } \\
\text {-L'industrie } \\
\text {-L'éducation }\end{array}$ \\
\hline Billet de 20 dinars type 1992 & $\begin{array}{l}\text { Kheireddine Pacha } \\
\text { (L'homme des réformes } \\
\text { sociales) }\end{array}$ & $\begin{array}{l}\text {-Les dômes de la mosquée de } \\
\text { "Sidi Mehrez" } \\
\text {-L'entrée de la chambre des } \\
\text { députés }\end{array}$ \\
\hline Billet de 5 dinars type 1993 & $\begin{array}{l}\text { Hannibal (Le chef guerrier, } \\
\text { le stratège) }\end{array}$ & $\begin{array}{l}\text {-Un épi de blé } \\
\text {-Le port militaire de l'amirauté } \\
\text { punique }\end{array}$ \\
\hline Billet de 10 dinars type 1994 & $\begin{array}{l}\text { Ibn Khaldoun (Le } \\
\text { sociologue) }\end{array}$ & -Ruelle de la Médina \\
\hline Billet de 30 dinars type 1997 & $\begin{array}{l}\text { Abou E1 Kacem Chebbi } \\
\text { (Le poète patriote) }\end{array}$ & $\begin{array}{l}\text {-Des ruines } \\
\text {-Une infrastructure composée } \\
\text { de routes } \\
\text {-Le planétarium de la cité des } \\
\text { sciences } \\
\text {-L'Institut National des } \\
\text { Sciences Appliquées de Tunis }\end{array}$ \\
\hline Billet de 10 dinars type 2005 & $\begin{array}{l}\text { Didon (La femme } \\
\text { émancipée) }\end{array}$ & $\begin{array}{l}\text {-Mosquée el Abidine } \\
\text {-Un motif typique à la } \\
\text { décoration des plafonds des } \\
\text { mosquées }\end{array}$ \\
\hline Billet de 50 dinars type 2009 & $\begin{array}{l}\text { Ibn Rachik El Kairaouani } \\
\text { (La culture, l'enracinement) }\end{array}$ & -La Cité de la culture \\
\hline
\end{tabular}

Les effigies de ces sept personnages illustres figurant dans la monnaie tunisienne, de 1956 jusqu'à 2010, font partie d'un tout c'est-à-dire qu'ils sont l'un des éléments constitutifs des billets de banque. On ne peut les extraire ou les mettre de côté pour les analyser car ils font partie d'un contexte formé par l'ensemble des éléments qui les entourent et avec qui, ils inter-réagissent obligatoirement. Le message ne peut être compris que si on prend en considération la totalité.

Or il s'est avéré qu'en analysant les billets de cette même période, on s'est aperçu qu'on a remémoré le patrimoine tunisien en même temps qu'on a mis 
en exergue différents signes de modernité. Certes, en plus des mosquées et des temples, on discerne d'autres établissements culturels tels que l'Institut National des Sciences Appliquées, la cité de la culture, etc. Ces édifices font partie de notre présent.

Ainsi, passé et présent cohabitent dans un même support. Ceci est la définition même de la modernité. « Dans le monde arabe au contraire, la modernité, ne vise pas à détruire les liens avec le passé mais à les rétablir après qu'ils aient été rompus par l'irruption de la culture occidentale ${ }^{9}$ ».Donc on ne peut être moderne que si on reconnaît notre passé. La modernité justifie qu'il y a évolution et progrès par rapport à un état précédent. La monnaie a illustré à sa manière notre patrimoine tout en le liant à sa manière aussi au développement de la Tunisie d'aujourd'hui. «Il s'agit bel et bien de notre passé, si riche de découvertes, de mutations, d'événements qui ont abouti à la constitution de l'être que nous sommes ${ }^{10} »$.

Cette dualité histoire/modernité relevée est une preuve supplémentaire que la monnaie tunisienne colle bien à l'histoire du pays, quoiqu'on ne puisse prétendre qu'elle raconte l'histoire : elle évoque juste certains épisodes importants de l'épopée du pays juxtaposés à sa continuelle marche de développement. Maintenant, si tout le travail de design fait avec et autour des personnages qui illustrent la monnaie se trouve justifié, et donne bel et bien une valeur ajoutée, il est logique et normal d'avancer qu'il en fait aussi un objet de musée.

\section{La monnaie objet de musée}

Les billets de banque et les pièces de monnaie sont des objets. "L'objet dans notre civilisation n'est guère naturel. On ne parlera pas d'une pierre, d'une grenouille ou d'un arbre comme un objet mais plutôt comme d'une chose. La pierre ne deviendra objet que promu au titre de presse-papier ${ }^{11}$ ", ceci veut dire qu'on ne peut considérer une chose en tant qu'objet que si elle est industrialisée. Or, on a évoqué auparavant qu'au sein de l'approche graphique (la chaîne graphique) les billets de banque passent par tout un processus d'impression. Donc ce sont des objets.

Le musée est un lieu où on conserve et on expose des objets de collection, des objets devenus rares. Ces objets ont une grande valeur historique pour le pays concerné. En effet, ils retracent son histoire tout en offrant des preuves tangibles des différentes époques passées, des fragments d'événements clé spécifiques à cette nation. Et c'est dans cette perspective qu'on peut insérer la monnaie comme objet de collection (classée sous le nom de « Numismatique ») et objet de musée.

9 MAMI KHARRAT Faouzia, "Les notions de "modernité" et de "patrimoine" dans le lettrisme arabe ", in Triki Samir (dir.), Les pratiques artistiques tunisiennes modernes et leur implication dans un réseau de concepts idéologiques, Unité de recherche : Pratiques artistiques modernes en Tunisie, 2008, p. 97.

10 Ibidem, p. 95.

11 MOLES Abraham, «Objet et communication », in Communications, n 13, 1969, p. 4. 
Toutes ces caractéristiques l'y habilitent, sa composition, son design, « ses discours » et connotations. Donc il est bien compréhensible de retrouver un musée qui garde et valorise cette monnaie témoin de son temps. Pour le musée de la monnaie de la Tunisie, on y trouve de la toute première monnaie jusqu'à celle d'aujourd'hui. Donc à travers la monnaie on peut voyager à travers l'histoire et replonger dans certaines époques.

Dans le cadre de cet article et comme on l'a énoncé précédemment, l'histoire est retracée grâce aux différents personnages illustres qui ont fait revivre toute une chronologie en commençant de l'antiquité jusqu'à arriver à l'époque moderne. Au sein de cette même histoire, on trouve une deuxième histoire relative à la succession de deux régimes politiques, dirigés par deux présidents de la République différents. Ces deux ères ont été marquées par le choix des personnages et l'agencement des différents éléments graphiques à l'intérieur d'un support rectangulaire qui est le billet de banque. C'est principalement à travers ce support que cette histoire est mise en valeur vu que les pièces de monnaie n'ont pas changé. C'est pour cette raison, entre autre, qu'on accorde autant d'attention à l'allure et à l'apparence de la monnaie, en dépit de sa fonction d'échange de biens et de services, car elle est aussi importante, dans la sauvegarde de l'histoire, que le thème évoqué.

\section{Discussion}

\section{Les apports de la recherche}

L'approche graphique a permis de parcourir les étapes de la création graphique des billets de banque. À partir d'un support déjà mis en circulation, on est parvenu à faire ressortir les gabarits de mise en page sur lesquels se sont basés les concepteurs ; ensuite, on les a comparés avec ceux des billets de quelques pays étrangers. Ceci a permis d'aboutir à une affirmation que toute la monnaie du monde se ressemble sur le plan formel et sur le plan de la composition et la gestion de la surface rectangulaire. L'approche sémiologique a abouti à une découverte des connotations et des messages cachés et dissimulés dans les billets de banque à travers l'analyse iconographique des différentes effigies (cadrage, angle de vue, posture, etc.). De plus si on ajoute à ces derniers les choix chromatiques ainsi que le reste des éléments iconographiques constituant les billets tels que les monuments et les édifices, on constate que chaque billet de banque raconte une histoire ou traduit un contexte qui le plus souvent est en relation directe ou indirecte avec la vie du personnage en question. La représentation de l'ensemble de ces figures historiques tend à faire revivre leurs souvenirs et à perdurer dans la mémoire des citoyens.

Le croisement de ces deux approches a justifié l'importance du design pour un tel support dont l'importance réside, essentiellement, dans sa valeur marchande et monétaire. 


\section{Limites et voies de recherche}

L'article s'est limité, uniquement, aux portraits figurant sur les billets de banque. On n'a pas pris en considération ceux présents dans les pièces de monnaie ou les médailles commémoratives. Il conviendra d'étendre la recherche sur ces deux dernières. De plus, la présente recherche s'est intéressée, essentiellement, aux personnages illustres et leurs fonctions et apports dans la monnaie tunisienne, elle peut se prolonger par un travail sur les monuments et édifices, qui eux aussi ont jalonné l'histoire moderne de la Tunisie.

Concernant l'approche graphique, on s'est focalisé surtout sur la composition du billet et la gestion de sa surface. Il serait aussi envisageable d'approfondir encore plus la recherche sur le dessin et la technique utilisée pour la représentation des effigies. De plus, on pourrait s'attarder sur les couleurs utilisées et l'analyse de leurs caractéristiques (teinte, saturation et luminosité). On a relevé des difficultés lors de la recherche des portraits originaux à partir desquels la représentation de quelques effigies a été faite.

Les méthodes ou les approches de travail étaient seulement graphiques et sémiologiques, elles pourraient être élargies à des études culturelles plus approfondies comportant et traitant d'autres domaines d'études, tels que la sociologie, la philosophie, la médiologie, etc.

Il serait aussi intéressant d'impliquer et de prendre l'avis des citoyens sur la monnaie qu'ils manipulent et qu'ils échangent entre eux, d'attirer leur attention sur ses caractéristiques graphiques, ses composantes, ses significations, etc. à travers des questionnaires par exemple. Essayer aussi de s'entretenir avec les dessinateurs et les concepteurs des billets et des pièces de monnaie afin de comprendre les motivations des choix qu'ils ont effectués ainsi que leurs sources d'inspiration.

\section{Conclusion}

Le but de cet article a été de comprendre pourquoi le côté esthétique, plus précisément à travers les effigies des personnages illustres, est si important dans la monnaie, en dépit de sa fonction d'échange qui a priori prime. La comparaison de quelques billets de différents pays, a permis de mieux comprendre l'univers de référence de la monnaie tunisienne. Les billets renfermant des effigies de personnages illustres, sont pensés et gérés presque de la même manière et obéissent aux même règles et critères graphiques (la composition du support, la technique utilisée pour le dessin, le cadrage, etc.). Le billet de banque, à travers le monde, est reconnu comme tel, indépendamment de sa valeur ou de sa provenance. Ceci nous amène à affirmer que toutes les monnaies du monde se ressemblent. Ce qui les différencie, c'est l'ensemble des choix des éléments graphiques mis en jeu et plus particulièrement le choix des personnages illustres, qui permettent à chaque billet de banque de se singulariser et de se distinguer. 
L'approche sémiologique a permis de montrer, dans le cas de la Tunisie, que les choix des personnages inclus dans les divers billets sont motivés par les connotations liées à ces célébrités. Des personnages "icônes " qui collent à l'histoire de la Tunisie. Chacune de ces effigies est mise dans un contexte bien particulier, composé de différents éléments iconographiques (des monuments, des édifices, des activités et des pratiques) qui interagissent en créant une dualité entre patrimoine et modernité, entre passé et présent, cette conception est d'une grande qualité historique et artistique certaine.

C'est ainsi qu'on peut affirmer que l'histoire même de la monnaie, porteuse des valeurs et de la culture du pays, se confond avec celle du pays, mais aussi avec l'évolution des tendances esthétiques et des moyens techniques. Cependant, cette histoire n'est pas racontée dans son ordre chronologique, sauf au cours de la présidence de Bourguiba. Les autres personnages ont été intégrés sans aucune suite «logique». En effet, l'histoire de la Tunisie, fourmille de personnages aussi importants que les sept figurants dans la monnaie courante et évoquant d'autres périodes historiques. On peut avancer que le choix ou la sélection qui s'est faite sur les personnages retenus et illustrés s'explique par les connotations qui en font des icônes nationales et qui les relient à des domaines bien particuliers eux-mêmes en relation directe avec l'évolution du pays. L'exemple du choix d'« Ibn Rachik El kairaouani» est édifiant. Il est représenté avec en arrière plan la nouvelle cité de la culture. Ce billet a été émis l'année où « Kairouan » était capitale culturelle.

La conception, telle que nous l'avons vu, de la monnaie est à l'origine de l'octroi de ses « lettres de noblesse » et de l'accès au «statut » d'objet de musée, la deuxième vie du billet de banque. Il est entendu que cette valeur prend toute son importance le jour où le billet n'aura aucune valeur monétaire, c'està-dire le jour où il sera retiré du marché, laissant émerger son côté esthétique.

\section{BIBLIOGRAPHIE}

\section{Dictionnaires}

CHEVALIER J. et GHEERBRANT A., Dictionnaire des symboles, Robert Laffont/ Jupiter, Paris, 1982, 1060 p.

\section{Ouvrages}

BOUZIRI Imen, « Le design comme facteur de modernisation a-t-il une influence sur le comportement du consommateur tunisien ? ", in Les formes utilitaires en Tunisie au $X X^{e}$ siècle / sous la Dir. de Filali Aicha, Unité de recherche : Pratiques artistiques modernes en Tunisie, 2008, 214 p.

COCULA B. et PEYROUTET C., Sémantique de l'image pour une approche méthodique des messages visuels, Delagrave, $232 \mathrm{p}$.

DUC, L'art de la composition et du cadrage : Peinture-Photographie-Bande DessinéePublicité, Fleurus Idées, Paris, 1993, 191 p. 


\section{C\&O n45}

KHIRI A. et ALEXANDROPOULOS J., Numismatique et histoire de la monnaie en Tunisie, Tome I : L'antiquité, Tunis, 2008, 335 p.

KHIRI A. et FENINA A., Numismatique et histoire de la monnaie en Tunisie, Tome III : La monnaie contemporaine, Tunis 2008, 279 p.

LANNOYE Vincent, L'bistoire de la monnaie pour comprendre l'économie, Le Cri Éditions, 2011, 426 p.

MAMI KHARRAT Faouzia, "Les notions de "modernité" et de "patrimoine" dans le lettrisme arabe ", in Triki Samir (dir.), Les pratiques artistiques tunisiennes modernes et leur implication dans un réseau de concepts idéologiques, Unité de recherche : Pratiques artistiques modernes en Tunisie, 2008, 254 p.

\section{Revues}

MOLES Abraham, "Objet et communication ", in Communications, n 13, 1969, $21 \mathrm{p}$.

\section{Mémoires}

BELHASSINE Rim, Le timbre-poste en Tunisie : approche historique et graphique, ISBAT, 2003.

GUIDARA Zouhour, L'image de la Tunisie indépendante à travers les billets de banque de 1956 à 1986, ISBAT, 2004.

Résumé : Cet article étudie la fonction des personnages illustres présents dans les billets de banque tunisiens de 1956 jusqu'à 2010 (fonction de différenciation vu que c'est l'élément graphique prépondérant de la composition) et leurs apports culturels de glorification du patrimoine tunisien. Les hypothèses sont vérifiées à travers deux approches graphique et sémiologique qui ont démontré l'utilité et l'importance du design (recherche de signes et de sens) pour les billets de banque. Cette utilité aura son importance ultérieurement lors de la deuxième vie de ces supports.

Mots-clés : personnages illustres, billets de banque, design, histoire.

Abstract: This article examines the function of renowned characters in the Tunisian bank notes from 1956 to 2010 (based on differentiation since it is the dominant graphic element of the composition) and their cultural contributions of glorification of Tunisian heritage. Assumptions are verified through two graphic and semiotic approaches that have demonstrated the usefulness and importance of design (looking for signs and meaning) for banknotes. This utility will be important later in the second life of these materials.

Keywords: renowned characters, bank notes, design, history. 